\title{
ICCPS Demo: Incident Management and Analysis Dashboard for Fire Departments
}

\author{
Geoffrey Pettet* \\ Vanderbilt University \\ Nashville, TN \\ geoffrey.a.pettet@vanderbilt.edu
}

\author{
Ayan Mukhopadhyay* \\ Vanderbilt University \\ Nashville, TN \\ ayan.mukhopadhyay@vanderbilt. \\ edu
}

\author{
Chinmaya Samal \\ Vanderbilt University \\ Nashville, TN \\ chinmaya.samal.1@vanderbilt.edu
}

\author{
Abhishek Dubey \\ Vanderbilt University \\ Nashville, TN \\ abhishek.dubey@vanderbilt.edu
}

\begin{abstract}
This work presents a dashboard tool that helps emergency responders analyze and manage spatial-temporal incidents like crime and traffic accidents. It uses state-of-the-art statistical models to learn incident probabilities based on factors such as prior incidents, time and weather. The dashboard can then present historic and predicted incident distributions. It also allows responders to analyze how moving or adding depots (stations for emergency responders) affects average response times, and can make dispatching recommendations based on heuristics. Broadly, it is a one-stop tool that helps responders visualize historical data as well as plan for and respond to incidents.
\end{abstract}

\section{CCS CONCEPTS}

- Mathematics of computing $\rightarrow$ Probabilistic representations; Markov processes; • Information systems $\rightarrow$ Decision support systems; Data stream mining; Spatial-temporal systems; • Applied computing $\rightarrow$ Decision analysis;

\section{KEYWORDS}

Streaming Survival Analysis, Decision Support System, EMS Dispatch

\section{ACM Reference format:}

Geoffrey Pettet ${ }^{*}$, Ayan Mukhopadhyay ${ }^{*}$, Chinmaya Samal, Abhishek Dubey, and Yevgeniy Vorobeychik. 2019. ICCPS Demo: Incident Management and Analysis Dashboard for Fire Departments. In Proceedings of 10th ACM/IEEE International Conference on Cyber-Physical Systems (with CPS-IoT Week 2019), Montreal, QC, Canada, April 16-18, 2019 (ICCPS '19), 2 pages.

https://doi.org/10.1145/3302509.3313329

\footnotetext{
* These authors had equal contribution in this work.

This work is sponsored by The National Science Foundation under award number CNS1640624.

Permission to make digital or hard copies of all or part of this work for personal or classroom use is granted without fee provided that copies are not made or distributed for profit or commercial advantage and that copies bear this notice and the full citation on the first page. Copyrights for components of this work owned by others than ACM must be honored. Abstracting with credit is permitted. To copy otherwise, or republish to post on servers or to redistribute to lists, requires prior specific permission and/or a fee. Request permissions from permissions@acm.org.

ICCPS '19, April 16-18, 2019, Montreal, QC, Canada

(C) 2019 Association for Computing Machinery.

ACM ISBN 978-1-4503-6285-6/19/04 . \$15.00

https://doi.org/10.1145/3302509.3313329
}

\author{
Yevgeniy Vorobeychik \\ Washington University \\ St. Louis, MO \\ yvorobeychik@wustl.edu
}

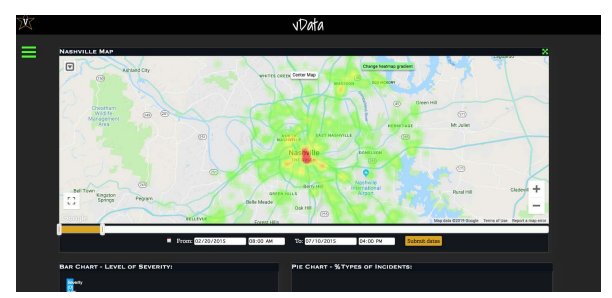

Figure 1: Historic Mode: Example of a heatmap produced for incidents occurring in Nashville between February 20th to July 10th, 2015

\section{PROJECT OVERVIEW}

The problem of stationing and dispatching emergency responders to service traffic accidents, fire, distress calls, and crimes plagues urban areas across the globe. While such problems have been extensively looked at, most approaches are offline, and such methodologies fail to capture the dynamically changing environments under which critical emergency response occurs. This is the primary reason why such methods are often not implemented in practice. Any holistic approach towards creating a pipeline for effective emergency response must also look at other challenges that it subsumes - predicting where and when incidents happen and understanding dynamic environments. We present a dashboard tool that collectively deals with these problems in an online manner, meaning that the models get updated with streaming data sources, while also allowing dispatchers to analyze past incident distributions and plan for future depot placement.

Our demonstration of this dashboard will include walking viewers through each of the dashboard's four features: historic incident analysis, future incident prediction, depot placement exploration, and dispatching. We will use past incident data from Nashville for the demo, since streaming data will be unavailable. The dispatch algorithm will be presented in detail in the conference [2].

\subsection{Statistical Foundation}

A fundamental necessity of any responder dispatch framework is to first understand when and where incidents happen. Formally, we want to learn a probability distribution over incident arrival in space and time. In order to do so, we leverage our prior work in which we have shown how survival models prove to be extremely effective in predicting incidents like crimes and traffic accidents 


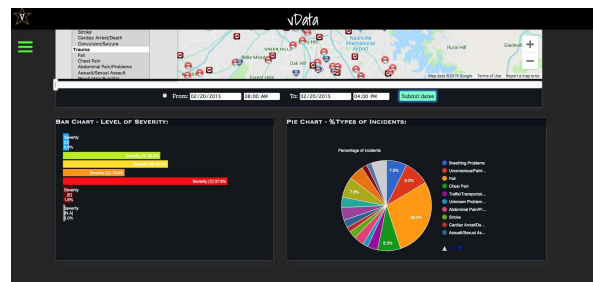

Figure 2: Historic Mode: The distribution of the severity and types of incidents occurring on February 20th, 2015

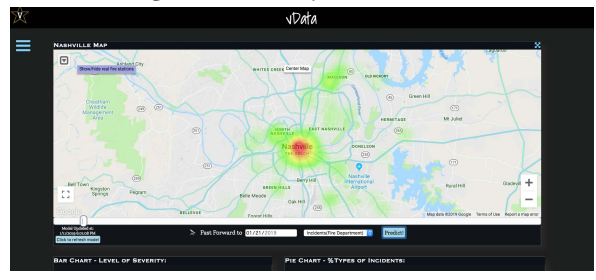

Figure 3: Prediction Mode: The incident distribution for January 21st, 2019 based on our prediction model

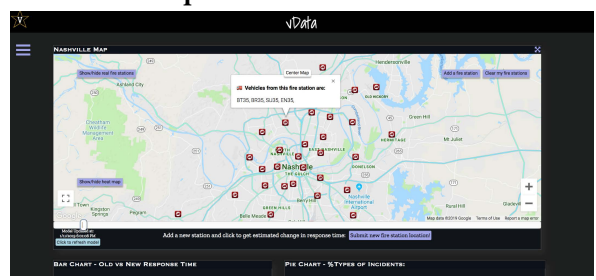

Figure 4: Explore Mode: The Locations of depots as displayed in the depot exploration mode. Each depot can be moved, and new depots can be added using the buttons in the upper right corner. The result on average response times is shown as a bar graph in the lower left

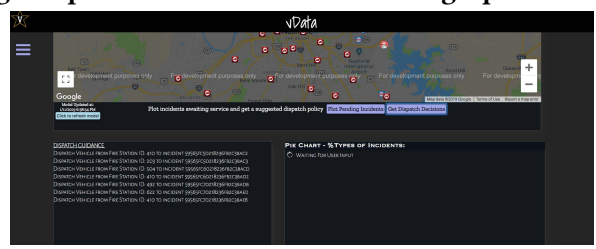

Figure 5: Dispatch Mode: Dispatch guidance given by the system recommending which vehicles should respond to pending incidents

[3-5]. Survival Analysis is a class of methods used to analyze data comprising of time between incidents of interest [1]. Survival models can be parametric or non-parametric in nature, with parametric models assuming that survival time follows a known distribution. Moreover, one may choose to model either the risk of occurrence of events (hazard) or the time to occurrence of incidents as the dependent variable of interest. We use a parametric model that lets us directly learn a distribution over inter-arrival time between incidents as a function of an arbitrary set of features (we use features such as prior incident data, weather and time of day). The resulting models have shown to outperform existing state-of-the-art in prediction accuracy [2], and lay the foundation for the dashboard's functionality.

\subsection{Demonstration}

We present an interactive web application providing responders with several views and functionality overlayed on a map interface.

- Historical Incident Density: The dashboard can display past historical incidents either plotted individually or as a heatmap, shown in figure 1. Users can select which dates they are interested in, so that they can examine particular times of interest. It also displays the types of incidents and their severity, shown in figure 2.

- Incident Density Prediction: Using our incident prediction model, the dashboard can display similar heatmaps for predicted future incidents, shown in figure 3. This allows responders to plan ahead for incidents likely to occur in the future.

- Depot Exploration: Cities are ever expanding and changing, so depot locations must be added and moved periodically. Using our incident model, our dashboard allows planners to see how specific depot positions affects average response times. Our map allows the user to interactively move and add stations, and then see their effect. This is shown in figure 4.

- Dispatch: The incident model can also be used to help make dispatching decisions. Our dashboard can see pending incidents, and makes recommendations about which responders to send to them (see figure 5). Most dispatchers currently simply send the closest responder, but this ignores future incident distributions [2]

Together these components come together to allow responders to analyze and properly plan for and respond to incidents.

\section{REFERENCES}

[1] David Roxbee Cox and David Oakes. 1984. Analysis of survival data. Vol. 21. CRC Press.

[2] Ayan Mukhopadhyay, Geoffrey Pettet, Chinmaya Samal, Abhishek Dubey, and Yevgeniy Vorobeychik. 2019. An Online Decision-Theoretic Pipeline for Responder Dispatch. In International Conference on Cyber-Physical Systems 2019. to appear.

[3] Ayan Mukhopadhyay, Yevgeniy Vorobeychik, Abhishek Dubey, and Gautam Biswas. 2017. Prioritized Allocation of Emergency Responders based on a Continuous-Time Incident Prediction Model. In International Conference on Autonomous Agents and MultiAgent Systems. International Foundation for $\mathrm{Au}^{-}$ tonomous Agents and Multiagent Systems, 168-177.

[4] Ayan Mukhopadhyay, Zilin Wang, and Yevgeniy Vorobeychik. 2018. A Decision Theoretic Framework for Emergency Responder Dispatch. In Proceedings of the 17th International Conference on Autonomous Agents and MultiAgent Systems, AAMAS 2018, Stockholm, Sweden, July 10-15, 2018. 588-596. http://dl.acm.org/ citation.cfm?id=3237471

[5] Geoffrey Pettet, Saideep Nannapaneni, Benjamin Stadnick, Abhishek Dubey, and Gautam Biswas. 2017. Incident Analysis and Prediction Using Clustering And Bayesian Network. In 2017 IEEE International Conference on Smart City Innovations. 